\title{
Rursus
}

Russus

Poiétique, réception et réécriture des textes antiques

$7 \mid 2012$

L'encyclopédie zoologique de Constantin VII

\section{Les panthères de Timothée de Gaza dans l'encyclopédie zoologique de Constantin VII}

Timotheus of Gaza's panthers in the zoological encyclopaedia of Constantine VII

Thierry Buquet

\section{(2) OpenEdition}

\section{Journals}

Édition électronique

URL : http://journals.openedition.org/rursus/971

DOI : $10.4000 /$ rursus. 971

ISSN : 1951-669X

Éditeur

Université Nice-Sophia Antipolis

Édition imprimée

Date de publication : 2 avril 2012

\section{Référence électronique}

Thierry Buquet, «Les panthères de Timothée de Gaza dans l'encyclopédie zoologique de

Constantin VII », Rursus [En ligne], 7 | 2012, mis en ligne le 25 avril 2012, consulté le 02 mai 2019.

URL : http://journals.openedition.org/rursus/971 ; DOI : 10.4000/rursus.971

Ce document a été généré automatiquement le 2 mai 2019.

Rursus 


\title{
Les panthères de Timothée de Gaza dans l'encyclopédie zoologique de Constantin VII
}

Timotheus of Gaza's panthers in the zoological encyclopaedia of Constantine VII

\author{
Thierry Buquet
}

\section{Introduction ${ }^{1}$}

$1 \quad$ L'œuvre de Timothée de Gaza, grammairien ayant vécu au tournant des $\mathrm{v}^{\mathrm{e}}$ et $\mathrm{vI}^{\mathrm{e}}$ siècles sous le règne de l'empereur Anastase ${ }^{2}$, est en grande partie perdue. Son traité zoologique, Peri zôôn, n'est connu, et encore de façon fragmentaire, qu'à travers des compilations médiévales byzantines et arabes. Des extraits de Timothée ont été inclus au $\mathrm{x}^{\mathrm{e}}$ siècle sous forme d'excerpta dans l'encyclopédie zoologique de Constantin VII, dite Syllogé Constantini (LAMBRos, 1885). Un épitomé anonyme byzantin, daté du XI ${ }^{\mathrm{e}}$ siècle, a été redécouvert au XIX e siècle et édité par Haupt (HAUPT, 1869) puis traduit en anglais par Bodenheimer et Rabinowitz (TIMOTHÉE DE GAZA, On animals, 1949)³. Enfin, Timothée de Gaza est utilisé dans plusieurs ouvrages de zoologie arabe : dans la partie zoologique de l'encyclopédie du $\mathrm{x}^{\mathrm{e}}$ siècle d'al-Tawhīdī (корғ, 1956) - mais de façon peu certaine ${ }^{4}$, et dans le traité zoologique (Ṭabā'i al-hayawān: Nature des animaux) de Marwazī (début du XII siècle), encore inédit (KRUK, 2001 ; ISKANDAR, 1981).

2 Timothée de Gaza était lu à l'époque byzantine et fut considéré comme une des autorités principales de la zoologie; Johannes Tzetzes (xiesiècle) le cite à ce titre au même rang qu'Oppien et Élien (sTEIER, 1937 : 1339). Le fait que Timothée soit paraphrasé ou abrégé aux $\mathrm{x}^{\mathrm{e}}$ et $\mathrm{xI}^{\mathrm{e}}$ siècles est un signe de la haute considération que l'on portait à son traité zoologique ; l'épitomé de Timothée devint si populaire qu'il remplaça l'œuvre originelle, ce qui pourrait expliquer ainsi sa perte (TIMOTHÉE DE GAZA, On animals : 7). 
3 L'étude de la transmission de Timothée de Gaza du $\mathrm{x}^{\mathrm{e}}$ au XII ${ }^{\mathrm{e}}$ siècle peut nous renseigner d'une part sur l'état du texte originel et d'autre part sur la lecture et l'utilisation de son œuvre dans les savoirs zoologiques médiévaux.

Dans le cadre de ce numéro consacré à la Syllogé Constantini, cet article se propose d'étudier un passage de l'encyclopédie zoologique de Constantin VII: les paragraphes 260 à 282, couvrant les pages 93 à 96 de l'édition de Lambros (LAMBROS, 1885 : 93-96). Selon l'éditeur, toute cette partie est empruntée directement à Timothée de Gaza, mais celui-ci n'y est pas cité nommément, contrairement à d'autres passages ${ }^{5}$. Les paragraphes précédents de l'épitomé ne comportent pas non plus d'indication de source et il faut attendre le $\S 283$ pour voir apparaitre ce type de mention (ici pour Aristophane de Byzance). Par la suite, ces mentions deviennent très fréquentes, sans être systématiques. Pour tous les passages non nommés, Lambros a proposé une attribution par la recherche des sources. De nombreux passages attribués ainsi à une compilation de Timothée ne reposent donc que sur la perspicacité de l'éditeur: ainsi les §193-197, 229-244, 260-282 et 588-589. Nous verrons, au long de cet article, si ces attributions sont toujours vérifiables pour les $§ 260-282$.

\section{Composition de l'extrait étudié}

5 Les paragraphes 260-282 traitent principalement de quatre animaux: pardalis (la panthère), kamelopardalis (la girafe), panthêr (le guépard), leopardos (le « léopard») au passage une digression sur la nature des hybrides et sur les unions constatées entre espèces différentes.

6 Les animaux cités dans ces passages entretiennent une relation étroite avec la panthère ( pardalis): soit ils la décrivent et racontent diverses anecdotes ou propriétés comportementales à son propos (\$ 260-269 et \$ 277), soit ils évoquent des animaux qui lui ressemblent (\$ 270-271 et § 278 pour la girafe ; § 274-276 pour le leopardos ; § 279-282 pour l'animal nommé panthêr). Au milieu, la digression sur les hybrides (§ 272-273) évoque une grande variété d'animaux, donnés à titre d'exemples (lion, panthère, tigre, ours, chien, renard, loup, hyène, antilope, cheval, bœuf).

7 Autant il peut sembler logique, selon notre regard moderne, de traiter ensemble des animaux qui se ressemblent ou peuvent être confondus (panthère, guépard, léopard), autant il peut paraître étrange de voir insérer ici la description de la girafe. Celle-ci intervient ici à titre d'exemple, en tant qu'animal paraissant être un mélange entre deux espèces, le chameau (kamelos) et la panthère (pardalis), mélange que l'on retrouve dans son nom (kamelo-pardalis), mais aussi par certains traits anatomiques évoquant ces deux espèces, notamment le pelage tacheté de la girafe qui peut évoquer celui de la panthère.

8 Après la digression sur les hybrides, le texte traite du leopardos, défini comme le produit de la lionne et de la panthère mâle, histoire bien connue depuis Pline l'Ancien (PLINE, Histoire naturelle, VIII, 17, 42-43 et 23,63), même si ce dernier ne cite pas le zoonyme leopardus. Pline raconte l'adultère entre la lionne et la panthère, histoire reprise par Timothée de Gaza.

9 Les paragraphes 280-282 traitant de la panthêr insistent aussi sur la nature hybride de cet animal, «hyper-hybride » même, car sa mère a recueilli les amours d'une multitude d'animaux pour le concevoir : l'hyène, la panthère, le lion, le loup et même, chose plus surprenante, le lièvre, le chamois et la gazelle! Timothée précise: «Son apparence le 
montre [son origine multiple], comme aussi son nom»: on retrouve ici l'étymologie fabuleuse de ce zoonyme : pan-ther signifie en grec « tout animal », ce qui permet à Isidore de Séville d'expliquer pourquoi la panthère est l'amie de tous les animaux (ISIDORE, Étymologies, XII, 2, 8) 7 .

Nous pouvons observer que tout le passage traite des panthères et de l'hybridité ; s'il fait suite à d'autres paragraphes traitant de la pardalis, ayant pour sources Aristophane de Byzance (\$ 245-251) et Élien (§ 252-259), il insiste quant à lui sur ces questions d'hybridité et décrit d'autres variétés de panthères, apportant des informations très utiles pour comprendre les confusions antiques et médiévales entre les grands félins, panthère, guépard et « léopard».

\section{La panthère (pardalis) : § $260-269$ et 277}

11 Au § 260, Timothée n'est pas cité nommément par le compilateur. Lambros a identifié ce passage à cause d'une remarque curieuse sur les deux sortes de panthères: les unes petites et les autres grandes; les petites panthères ont une grande queue et les grandes une petite queue (LAMBROS, $1885: 93)^{8}$. Ce détail anatomique et l'évocation de deux sortes de panthères (pardalis) n'existe qu'à deux reprises dans la littérature antique: chez Timothée de Gaza (On animals, §11, p. 25) et chez Oppien (Cynégétiques III, 63: trad. L'Allier, 2009: 95). Dans l'épitomé du $\mathrm{xl}^{\mathrm{e}}$ siècle, le texte présente des lacunes dans l'édition de Haupt et la traduction de Bodenheimer, et les éditeurs ont pu reconstituer ce passage à partir d'Oppien; cette reconstitution est ainsi confirmée par l'excerpta plus détaillé de la Syllogé Constantini. De plus, ce passage est également repris dans les mêmes termes par Marwazī et sa source attribuée à Timothée par Remke Kruk (кRUK, 2001 : 369) ${ }^{9}$, donnant là une confirmation supplémentaire de la reconstitution du passage de Timothée. Mais il faut préciser que Marwazī ne cite pas nommément ici Timothée et ne donne aucun autre élément relatif à sa description de la panthère. Marwazì évoque le guépard (arabe : $f a h d)^{10}$ et non pas la panthère, et continue ensuite par la description du dressage du guépard comme auxiliaire de chasse, technique inconnue des sources grécolatines jusqu'au début du $\mathrm{VI}^{\mathrm{e}}$ siècle (VINCENT,1994) ${ }^{11}$. Dans la tradition textuelle cynégétique arabe, la question des deux variétés de panthères à la queue longue ou courte se retrouve dans d'autres ouvrages : citons le traité de chasse avec des animaux et des oiseaux de proie (Kitab al-mașāyid wa'l-mațārid, éd. TALAS, 1954 : 211-212) de Kušǎğim (m. avant 971) et dans le livre de chasse d'al-Mansūr, daté du XIII ${ }^{e}$ siècle (AL-MANSŪR, éd. CLARK \& DERHALLI, 2001: 76) ${ }^{12}$. Le contexte cynégétique suggérerait plutôt une citation directe d'Oppien dans ces deux cas; on peut émettre l'hypothèse que Marwazī utilise cette tradition cynégétique gréco-arabe plutôt que le texte de Timothée de Gaza. Il est donc sans doute abusif de voir dans le passage cité de Marwazī un écho de l'œuvre de Timothée de Gaza; dans ce cas, c'est bel et bien le texte de la Syllogé Constantini et lui seul qui permet de retrouver un état plus complet du Peri zôôn de Timothée.

Les paragraphes suivants ( $\$ 261-269$ ) mélangent des informations très diverses provenant de la mythologie grecque ( $\$ 266$ : Penthée transformant les nourrices de Dyonisos en panthères), de légendes anciennes, reprises chez les Autorités ou dans le Physiologos (amour du vin, dard au bout de la queue, langue rugueuse comme celle du lion, ruses de chasse, peur du feu) et d'après l'observation (couleurs et taches du pelage, \$269). Toutes ces données hétérogènes sont faciles à attribuer à Timothée, car on retrouve presque tous 
ces éléments, dans un ordre légèrement différent, dans l'épitomé du $\mathrm{XI}^{\mathrm{e}}$ siècle ( TIMOTHÉE DE GAZA, On animals : 25, 11). Seuls y manquent la peur du bois de cornouiller et les variations de couleurs du pelage ( $(268-269)$.

Dans le $\$ 265$ est décrite une ruse utilisée par les vieilles panthères (pardalis) qui, ne pouvant plus chasser, dressent un piège aux chèvres sauvages : par le dépôt d'excrément et d'urine à un carrefour, elles forcent leurs proies à se diriger vers elles ${ }^{13}$. Ce qui est remarquable, c'est qu'ici la pardalis piège les animaux avec ses mauvaises odeurs, alors que la panthêr indienne les attire par la douceur de son haleine (\$282) ${ }^{14}$ !

Dans ces paragraphes sur la pardalis, Timothée de Gaza ne fait guère montre d'originalité ou d'observation zoologique poussée : il semble reprendre presque tout son savoir à celui des Autorités, en premier lieu Oppien, en y mêlant tout un folklore légendaire et mythologique. Seul le dernier paragraphe (\$269) portant sur les divers types de pelage (du blanc au noir en passant par le jaune) semble original dans la littérature antique et a quelque fond de vérité zoologique. Si l'existence de panthères noires est bien connue ${ }^{15}$, les diverses sous-espèces de panthère présentent des variétés de couleurs importantes selon l'habitat, allant d'un jaune très pâle au roux plus marqué, sans oublier le blanc pour la panthère des neiges (Uncia uncia). Il en est de même pour le guépard, auquel le grec pardalis peut être parfois identifié (voir plus loin). Nous en avons un très bon témoignage dans le traité de la chasse d'Ibn Manglī (xIve siècle) qui, dans sa description des différentes variétés de guépards asiatiques ${ }^{16}$, évoque le roux franc, le blanc, le jaune et même le noir pour ceux originaires du Yémen (IBN MANGLĪ, De la chasse, trad. Viré, 1984 : 99).

\section{La girafe : § $270-271$ et 278}

Suivant immédiatement le paragraphe décrivant les différentes couleurs des robes des panthères (pardalis), la girafe (kamelopardalis) est présentée comme une sorte de mélange

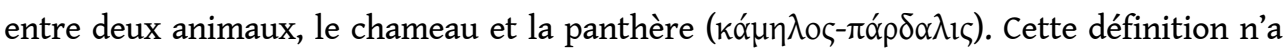
rien d'étonnant, car elle se retrouve dans la plupart des textes grecs et latins décrivant la girafe depuis les premiers témoignages datant de la période hellénistique (GATIER, 1996). La transition panthère-girafe à cet endroit dans l'encyclopédie peut s'expliquer par la ressemblance supposée du pelage entre les deux animaux; dans l'épitomé, le paragraphe décrivant la girafe ne suit pas immédiatement celui évoquant la panthère et en est assez éloigné (chap. 24 pour la girafe, 11 pour la pardalis). L'identification de la source des \$ 270-271 vient d'une anecdote très précise et circonstanciée : l'auteur évoque le passage dans sa ville de Gaza de l'animal qui devait être amené à l'empereur Anastase. La mention de Gaza et la période du règne d'Anastase identifient à coup sûr Timothée de Gaza, d'autant que l'épitomé reprend aussi ces informations. Cette anecdote permet de confirmer les éléments de la biographie de Timothée donnés par Suidas, qui le place durant le règne d'Anastase. Le cadeau de deux girafes, offertes à l'empereur Anastase et accompagnées d'un éléphant ${ }^{17}$, est également évoqué par la Chronique de Marcellin pour l'année 496 (MARCELLINUS COMES, éd. MOMMSEN, 1894: a 496, p. 94) ${ }^{18}$. Il s'agit sans aucun doute du même événement évoqué dans les compilations de Timothée, le chiffre de deux girafes étant identique dans chaque texte. Marwazī, qui cite là nommément Timothée, est beaucoup plus précis : les deux spécimens ont été amenés à l'auteur dans sa maison, et Timothée a pu ainsi les observer et faire part de son émerveillement face à leur forme et leur nature. Marwazì confirme le statut d'animal d'apparat de ces girafes, qui sont 
couvertes de vêtements et tenues par des brides et des colliers nasaux. Il ajoute aussi que les bêtes étaient montées par des hommes noirs (KRUK, 2001 : 364) ${ }^{19}$. Marwazī complète ici ce qui reste de la notice de Timothée dans l'excerpta de la Syllogé et dans l'épitomé et confirme que Timothée a été un témoin direct du passage des animaux envoyés à Anastase. La Syllogé Constantini et l'épitomé diffèrent légèrement dans le passage relatif au personnage qui accompagnait les animaux : dans le premier texte, l'homme est «indien » alors que dans le second il est originaire d'Aila et apporte des marchandises indiennes ${ }^{20}$. Le second épitomateur a sans doute mal compris le texte original de Timothée qui devait indiquer que le convoi provenait d'Aila ${ }^{21}$, accompagné par un "Indien». Marwazī, à l'instar de la Syllogé Constantini, ne parle pas d'Aila, mais signale que l'homme, originaire de l'Inde, était messager du roi de ce pays (KRUK, $2001: 364$ ). Ici les trois témoins de l'œuvre perdue de Timothée apportent des détails différents et complémentaires, et on peut reconstituer ainsi la teneur de ce passage dans le traité original: un indien originaire de l'Inde et messager du souverain de ce pays passa à Gaza avec deux girafes destinées à l'empereur Anastase, en provenance de la ville d'Aila, d'où il avait sans doute débarqué après un voyage maritime depuis le Sud.

Le passage des girafes à Gaza aurait contribué à créer une tradition iconographique dans cette région, où de nombreuses mosaïques représentent des convois venus d'Orient amenant des girafes, des éléphants, des zèbres et des autruches (GATIER, 2005). La présence de zèbres sur ces mosaïques pourrait suggérer que cet animal, sans doute très rare dans l'Antiquité, se trouvait peut-être parmi les cadeaux envoyés à Anastase et expliquerait alors que Timothée de Gaza en ait eu connaissance et en donne une description, évoquée rapidement dans l'épitomé du $\mathrm{xl}^{\mathrm{e}}$ siècle (voir plus loin). Mais Timothée ne parle pas ici d'Anastase et évoque au contraire les jeux du cirque à Rome, où les zèbres, au sein d'une procession, conduisaient des chariots qui transportaient la princesse impériale ${ }^{22}$.

17 La compilation de Marwazì du passage de Timothée sur la girafe semble confirmer, pour reprendre les conclusions de Remke Kruk, que cet auteur a eu accès au XII siècle à une version plus complète de l'œuvre de Timothée (KRUK, 2001:364,384), peut-être par l'intermédiaire de traductions arabes qui ont circulé à l'époque médiévale (KRUK, 2001 : 358).

18 Le $\$ 272$ offre des informations intéressantes sur la démarche de la girafe qui va l'amble: le compilateur écrit que son allure n'est semblable à celle d'aucun autre animal, car elle lance en même temps les deux pieds du même côté. Cette information est présente aussi chez Marwazī, confirmant l'autorité de Timothée. Il faut préciser qu'un seul auteur antique décrit aussi précisément l'allure de l'animal, Héliodore (Éthiopiques, 10, 27-28, trad. MAILLON, 1943 : t. III, 108-109), qui utilise à peu près les mêmes termes que Timothée, ce dernier lui ayant peut-être emprunté ce détail. Oppien évoque également cette démarche particulière, mais son texte n'a pas la clarté et la précision d'Héliodore ou de Timothée et a toujours posé des problèmes aux traducteurs ${ }^{23}$. Oppien évoque une démarche boiteuse qui serait due à la différence de taille entre les antérieures et les postérieures, sans doute n'avait-il pas bien perçu que cette impression de claudication était due à l'amble. Par la suite, Michel Attaleiatès (Histoire, 29, éd. GRÉGOIRE, 1958: 357-358) et Michel Psellos (Orationes panegyricae, I, 278-289, éd. DENNIS, 1994 : 13-14) vont donner de bonnes descriptions de l'amble, sans qu'il soit facile de savoir si elles 
proviennent de l'observation du spécimen de Constantin IX au XI ${ }^{e}$ siècle, ou si ce détail anatomique a pu être repris de Timothée ou d'Héliodore.

Le passage sur l'amble dans la Syllogé Constantini a été commenté par Morgan (1988)

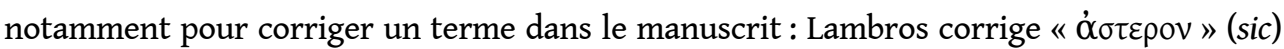

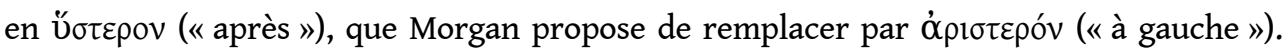
Morgan explique que le terme choisi par Lambros est une erreur au regard de tout le règne animal : en effet le texte de Lambros donne :

... ceux-là lancent à tour de rôle de chaque côté le pied, du côté droit le pied avant, puis ensuite celui de derrière...

\section{La correction proposée par Morgan donne :}

...ceux-là lancent à tout de rôle de chaque côté le pied, du côté droit le pied avant, puis à gauche celui de derrière...

21 Ce qui correspond bien à la réalité de la marche des quadrupèdes, telle qu'elle est décrite par Aristote (De incessu animalium, 712 a $25 \mathrm{ss}$ ), qui meuvent la jambe avant droite puis l'arrière gauche, puis l'avant gauche et enfin l'arrière droite a droite de derrière (Morgan 1988 : 268).

22 La description de la girafe par Timothée semble peu influencée par celle d'Oppien ${ }^{24}$, d'une part sa description de l'amble est beaucoup plus précise, d'autre part Timothée ne décrit pas les cornes de la girafe dans ce passage. Les détails anatomiques décrits par Marwazī et empruntés à Timothée se démarquent de façon significative de la brève notice d'Oppien.

Le deuxième passage (\$ 278) évoquant la girafe, attribué à Timothée par Lambros, me semble plutôt faire référence à Philostorge, qui est le seul auteur antique à comparer la girafe avec le cerf : si Oppien dit qu'elle a la même bouche que le cerf, Philostorge écrit que c'est la forme générale de son corps qui évoque cet animal ${ }^{25}$. Tous les éléments de la description de Philostorge (Histoire ecclésiastique, III, 11, éd. BIDEZ, 1913 : 40-41), corps de cerf, stature du chameau, cou très haut et très long, hauteur des antérieures, se retrouvent dans la Syllogé Constantini. Il n'y a qu'un seul détail qui ne se trouve pas dans la notice de Philostorge : les cornes de la girafe, ou plutôt son absence, selon le texte de la Syllogé. Dans la description de la girafe de Marwazī, on ne trouve pas de comparaison avec le cerf, et il n'est pas fait mention des cornes (KRUK, 2001:364). Le texte de Marwazī a d'évidents points communs avec le premier passage de l'encyclopédie (270-271) et aucun avec le second (278). Tout porte donc à croire que le \$ 278 est une citation de Philostorge par le rédacteur de l'encyclopédie. Une seule mention de Philostorge a été repérée par Lambros dans son édition ( $\$ 132$, p. 71-72): un passage du livre III, 11 de l'Histoire ecclésiastique (d'où provient le passage sur la girafe) relatif aux éléphants et aux taurelephantos ${ }^{26}$. Il faudrait sans doute réévaluer l'utilisation de Philostorge dans la Syllogé Constantini et vérifier si d'autres emprunts à son œuvre sont repérables, mais ceci sort du cadre de notre étude.

24 La question des cornes de la girafe est intéressante à plus d'un titre. Un seul auteur antique les mentionne : Oppien, dans ses Cynégétiques, qui explique que ce ne sont pas des

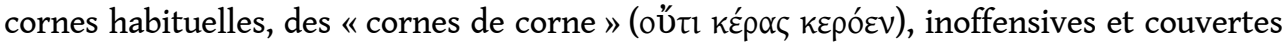
de poils (oppIEN, Cynégétiques, III, v. 475, éd. MAIR, 2002 : 152-153). Par la suite, aucun auteur byzantin n'a décrit ces cornes, y compris les chroniqueurs ayant pu observer les spécimens amenés à Byzance aux XI ${ }^{\mathrm{e}}$ et XIII ${ }^{\mathrm{e}}$ siècles. Aucune encyclopédie médiévale ne traite de cette question, et seuls quelques voyageurs occidentaux en Égypte les mentionnent à partir de la fin du XIV siècle. Il faudra attendre les humanistes du $\mathrm{Xv}^{\mathrm{e}}$ 
siècle pour avoir de meilleures descriptions des cornes de la girafe et notamment celles de Pierre Gilles d'Albi, André Thevet et Prosper Alpin pour savoir qu'il existe aussi une troisième corne frontale. Bien plus tard,Buffon consacrera plusieurs pages à cette question (BUFFON, 1765a:12-13), discutant les opinions des auteurs qui l'ont précédé, notamment les érudits et voyageurs; puis il renouvellera son information dans un chapitre entier du Supplément à l'Histoire naturelle consacré à la nature des cornes de la girafe, pour tenter de savoir si elles sont de la nature des bois de cerfs (et discutant la question de leur possible caducité), si elles sont bien différentes des cornes creuses des bovidés, voire de la nature de celles des rhinocéros, en poils (BUFFON, 1765b : 320-330).

Oublier de décrire ces cornes qui ne ressemblent pas aux cornes pointues des bovidés est une chose compréhensible, d'autant que chez les jeunes girafons, les cornes sont encore molles et pas encore complètement soudées à l'os crânien. Certains chercheurs se sont d'ailleurs demandés si les girafes vues en captivité dans les cours royales ou impériales antiques n'étaient pas la plupart du temps de jeunes animaux, récemment capturés en Afrique (CANNUYER, 2010: 167-184), car on avait l'habitude de tuer la mère pour les attraper (BUQUET 2012a: 71, note 37). Au XIII ${ }^{\mathrm{e}}$ siècle, Georges Pachymérès affirme que la girafe n'a pas de cornes. Il semble décrire un animal de petite taille, grand comme un âne canthon (Pachymères, Relations historiques, III, 4, éd. FAILLER, 1984 : 238-239). De son côté, le compilateur de la Syllogé Constantini, qui ne cite pas les cornes, ne semble pas avoir vérifié leur description chez Oppien, ou peut-être a-t-il compris dans ce passage des Cynégétiques que la girafe n'avait pas de vraies cornes...

Le passage de Marwazī sur la girafe de Timothée ne mentionne pas non plus les cornes, et ne présente pas la même structure sur le passage de la Syllogé emprunté à Philostorge, preuve supplémentaire que le $\S 278$ n'est pas repris à Timothée. Le texte de Marwazī a de nombreux points communs avec le $\$ 272$ de la Syllogé: queue de la gazelle, stature du chameau, etc.

L'épitomateur $\mathrm{du} \mathrm{XI}^{\mathrm{e}}$ siècle ajoute une remarque personnelle à l'abrégé de la notice de Timothée sur la girafe : girafe et éléphant ont été vus à son époque, sous le règne de Constantin IX Monomaque, amenés également de l' "Inde», et exhibés comme des merveilles dans le théâtre de Constantinople. Cette remarque permet de dater très précisément l'épitomé au XI ${ }^{\mathrm{e}}$ siècle, sous le règne de Constantin (1042-1055). Un glosateur du XI ${ }^{\mathrm{e}}$ siècle sur Agatharchides (MÜLLER, $1855:$ 159, fr. 72), en commentant un passage sur la girafe, dit qu'il a vu l'animal, envoyé par « le prince d'Alexandrie » (GATIER, 1996 : 926). Michel Attaliatès, dans sa chronique du règne de l'empereur Constantin IX, donne une bonne description de la girafe, arrivée à Byzance en 1053 (ATTALEIATÈs, Histoire, 29, éd. GRÉGOIRE, 1958 : 357-358). Comme le glosateur anonyme à Agatharchidès, il précise que la girafe venait d'Égypte, ce qui est également confirmé dans les Annales de Michel Glykas ( GLYKAS, Annales, IV, éd. BEKKER, $1836: 597,1.13-14)^{27}$. Attaliatès est également l'un des rares auteurs médiévaux à mentionner la marche à l'amble de la girafe, preuve de son grand sens de l'observation ou de son érudition : peut-être a-t-il emprunté ce passage à Timothée ou à Héliodore.

\section{Hybridations : $§ 272-273$}

Les paragraphes 272 et 273 ne constituent pas une notice sur un animal particulier mais rassemblent des considérations diverses sur la nature des animaux hybrides. Au § 272, il 
est question de la ressemblance des hybrides avec leur mère, avec l'exemple d'une femme dont le petit fils naquit avec la peau noire, trahissant les amours de sa mère avec un Éthiopien. Au § 273, le texte donne de nombreux exemples d'unions entre espèces très variées : lion-panthère, lion-chien, loup-hyène, antilope-cheval, renard-chien, ours-chien, etc. Parmi ces exemples, est évoquée l'union entre des tigres et des chevaux (\$ 273, 1. 9-10, p. 95). Il faut d'abord noter que la Syllogé Constantini ne comporte pas de notice sur le tigre, animal bien connu dans l'Antiquité, notamment décrit par Oppien et surtout par Timothée, dont un résumé de la notice est conservé dans l'épitomé (тімотнÉE DE GAZA, On animals, chap. 9 : 23-24) et par Marwazī (кRUK, 2001 : 368-369). Ensuite, chez Timothée ( тімотнÉE DE GAZA, On animals, chap. $10: 25)$ figure une description du zèbre (hippotigris, iđtótıүрıc), là aussi non reprise dans la Syllogé Constantini. Il s'agit pourtant de l'une des seules descriptions antiques du zèbre dont nous disposons: seulement deux autres

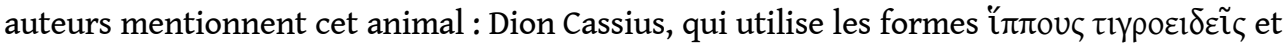

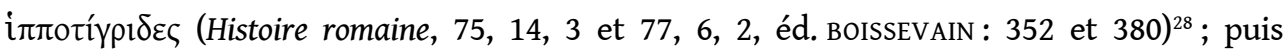
Philostorge (Histoire ecclésiastique, 3, 11), qui décrit parfaitement le zèbre et ses rayures, mais le nomme onos agrios, « âne sauvage $»^{29}$.

Dans l'épitomé de Timothée du $\mathrm{xI}^{\mathrm{e}}$ siècle, le paragraphe sur l'hippotigris n'a pas de rubrique, ce qui pourrait expliquer en partie son absence dans la compilation de la Syllogé Constantini. Le compilateur avait dû pourtant repérer l'hippotigris chez Timothée ou chez Philostorge car il signale que le tigre (tigris) et le cheval (hippos) peuvent s'accoupler (\$ 273, 1. 9-10, p. 95). Au final, on peut s'interroger sur les motivations du compilateur de la Syllogé, qui supprime les notices d'animaux aussi rares et curieux que le zèbre ou le tigre; on voit par là qu'il ne se sent pas lié à ses sources et sélectionne ou omet ce qui l'intéresse.

\section{Le léopard : § 274-276}

En suivant les paragraphes précédents sur l'hybridité, la Syllogé donne un exemple plus

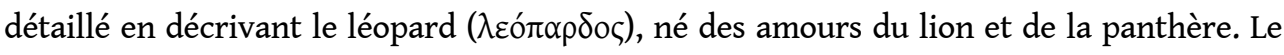
léopard hérite ainsi du pelage tacheté de la panthère, du courage et de la force du lion. En 275-276, la lionne doit cacher le petit issu de son union avec la panthère, car il porte, par ses taches, la preuve de l'adultère de sa mère ; celle-ci doit se laver pour effacer l'odeur de la panthère et ainsi éviter que le lion ne la tue ainsi que son petit. Cette histoire est bien connue et est racontée par Pline (Histoire naturelle, VIII, 17, p. 42-43), à la différence que celui-ci ne nomme pas le fruit de cette union. En effet, les mots leopardus ou leopardos ne sont attestés pour la première fois qu'au $\mathrm{II}^{\mathrm{e}}$ siècle chez Ignace d'Antioche ${ }^{30}$ puis dans $\mathrm{l}^{\prime}$ Histoire Auguste (Héliogabale, 21, 1 : éd. TURCAN : 99-100 et 25, $5: 103)^{31}$. Il serait vain de tenter d'identifier quelle espèce se cache derrière ce leopardos sous la plume de Timothée. En Occident médiéval, le mot leopardus peut désigner à la fois la panthère et le guépard et le plus souvent ce dernier dans un contexte vernaculaire. Par exemple, à la cour de l'empereur Frédéric II, il est question de leopardi dressés pour la chasse (BUQUET, 2011 : 26). Les choses sont encore plus complexes dans le monde byzantin. Le guépard, quand il peut être formellement identifié dans les textes, est la plupart du temps appelé pardus (comme chez Luxorius, voir supra) pardos ou pardalis mais jamais, semble-t-il, leopardos ( NICHOLAS, 1999 : 257-262). Les quelques mentions d'importation de leopardi dans les textes byzantins ne permettent pas d'affirmer s'il s'agit de panthères ou de guépards. Par exemple, à l'époque de Justinien, pour l'année 533, on taxait l'importation des pardi, 
leopardi et pantherae, sans qu'il soit possible de traduire ces trois termes avec certitude. Il est même difficile de savoir si la liste propose des termes synonymes pour un même animal ou si, dans l'esprit du législateur, trois espèces bien différenciées étaient concernées par les taxes d'importation (MOMMSEN \& KRUGER, 1889 : vol. 1, 606).

Dans la littérature byzantine, le mot leopardos ne semble renvoyer à aucune réalité zoologique : c'est un être mixte, qui n'apparaît qu'en tant qu'animal fabuleux, n'ayant pour propriétés que son hybridité et sa bâtardise, comme, au XIV siècle, dans le conte

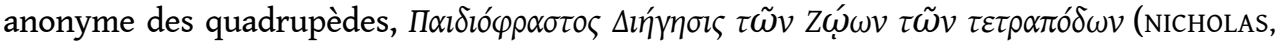
1999 : 279 ; NICHOLAS \& BALOGLOU, 2003).

\section{Le guépard : $§ 277$ et $279-282$}

Au $\$ 277$, il est dit que la pardalis est impétueuse et irascible et son corps souple et léger s'accorde à son tempérament. Ces remarques sont absentes de l'épitomé du XI ${ }^{\mathrm{e}}$ siècle, et le fait que ce paragraphe ne suive pas les précédents sur la pardalis dans la Syllogé pourrait indiquer une autre source que Timothée de Gaza. Aucune indication n'a été trouvée non plus chez Marwazì. La mention du caractère impétueux de la pardalis pourrait provenir d'un traité de physiognomonie, comme le suggère Lambros en note $(1.26$, p. 95) en citant le traité du pseudo-Aristote (Physiognomonie 5,809 b. 36 - $810 \mathrm{a} 10$ ) à propos de l'adjectif @ $\alpha \gamma \delta \alpha$ ĩov (impétueux, véhément). Dans les traités de physiognomonie antique, tout porte à croire que la pardalis ne désigne pas la panthère mais le guépard (ZUCKER, 2006: § 19, note 53). Ceci est particulièrement frappant dans le cas du pseudo-Aristote ( Physiognomonie, $809 \mathrm{~b} 40 \mathrm{sq}$.) qui décrit la pardalis de la façon suivante : petite face, large gueule, petits yeux plutôt pâles, enfoncés et très mobiles, front allongé, plutôt arrondi que plat au niveau des oreilles, cou long et fin, poitrine étroite, dos long, croupe et cuisses charnues mais flancs et ventre plats; couleur bariolée, ensemble désarticulé et mal proportionné (ZUCKER, 2006 : note 53). La description du cou du guépard se trouve dans le $\S 279$ de la Syllogé (mais cette fois sous le nom de panthêr): "Le guépard (panthêr) ressemble à la panthère (pardalis), à ceci près qu'il a un petit cou, une face $\operatorname{arrondie}^{32}$, et qu'il ne remue pas l'extrémité de sa queue ». Le texte édité par Lambros dit " petit cou » (

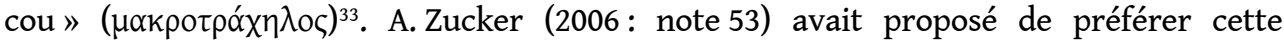

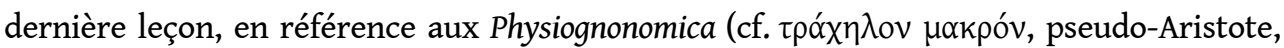
Physiognomonie, 810 a 3). Cela est confirmé par certaines différences anatomiques entre la panthère (cou trapu, épais et puissant) et le guépard (cou mince et plus allongé, tête plus petite et plus ronde).

L'origine hybride supposée de la panthêr donne lieu à une composition fantastique regroupant des parties de différents animaux, formant un véritable puzzle zoologique (\$ 281) : l'animal ainsi composé a un front d'hyène, la gueule de la pardalis, les cornes du

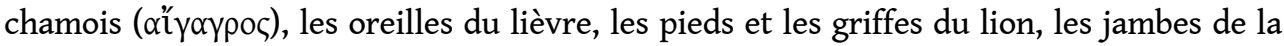
gazelle, le poitrail du lion et de la pardalis, la queue du loup et la peau tachetée de la pardalis. Dans l'épitomé du $\mathrm{xI}^{\mathrm{e}}$ siècle, il est simplement dit que la mère a conçu cet animal avec le plus grand nombre d'espèces possibles (TIMOTHÉE DE GAZA, On animals, $14: 26$ ). Chez Marwazī, on retrouve la majeure partie de ces comparaisons : jambes de gazelle, taches de la panthère, front de l'hyène, poitrail du lion, queue du loup (KRUK, 2001:374), avec de légères différences avec la description de l'encyclopédie zoologique de Constantin : tête 
de l'hyène, yeux et gueule du lion, pattes et hanches de la gazelle, poitrine et reste du corps du guépard (ar. fahd) et du lion, peau tachetée de la panthère (ar. namir). Il faut

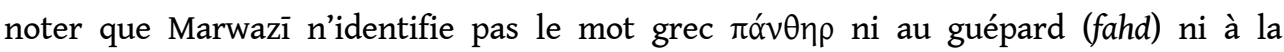
panthère (namir). Marwazī précise que le zoonyme est grec: «A beast of prey that is called in Greek (rūmìya) *bantīr » (KRUK, 2001 : 373-374), et ne le comprend pas. Ailleurs, il avait traduit pardalis en fahd " guépard» (KRUK, $2001: 369$ ), preuve que, pour un auteur arabe de ce temps pourtant au fait des différences entre panthère et guépard ${ }^{34}$, le lexique grec des grands félins reste obscur. Les textes antiques grecs et latins divergent selon les auteurs quant à l'interprétation à donner aux zoonymes pardalis, pardos, leopardos, panthera, panthêr(WOTKE \& JEREH, 1949 : col. 747-778). Une étude reste à faire pour faire le tour de la question, en y incluant une problématique temporelle, le sens des mots ayant $\mathrm{pu}$ évoluer des premières mentions des époques classique et hellénistique jusqu'à l'Antiquité tardive ${ }^{35}$. Dans la Syllogé, le terme đóv $\theta \eta \rho$ désigne certainement le guépard et c'est là, comme nous l'avons vu, un héritage des textes antiques ${ }^{36}$. À Byzance le guépard est connu, semble-t-il, depuis l'Antiquité tardive, à la fois dans les représentations et dans les textes. Dans les sources ayant trait à des réalités vernaculaires (chroniques, traités

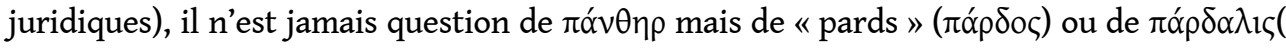
NICHOLAS, 1999). La description par Constantin Pantechnès d'une chasse au XII ${ }^{\mathrm{e}}$ siècle, utilisant comme auxiliaire des "pardalis » $(\pi \alpha \rho \delta \alpha ́ \lambda \varepsilon 1 \zeta)$ dressées à capturer des lièvres ( NICHOLAS, 1999, Appendix : 289-296), évoque de façon certaine les techniques de chasse au guépard bien connues en Inde, en Iran et dans le Proche-Orient arabo-musulman (VIRÉ , 1977).

Les éléments les plus surprenants de la notice de la Syllogé, les cornes du chamois et les oreilles du lièvre, sont absents du texte de Marwazī. Et pour cause, serait-on tenté de dire, car leur présence y semble fautive, en rapport aux autres détails anatomiques, dont la comparaison avec les autres animaux cités est plausible, comme la finesse et la célérité des pattes du guépard évoquant celles de la gazelle. Si on garde l'hypothèse de la proximité de la compilation de Marwazī avec un original perdu de Timothée, on peut considérer la présence du chamois et du lièvre comme un ajout du rédacteur byzantin de l'encyclopédie. Mais comment peut-il se motiver? Le compilateur n'avait peut-être jamais vu de panthère ou de guépard, au contraire de Marwazì, sinon il n'aurait peut-être pas ajouté ses détails fantaisistes. Faute de trouver une explication rationnelle, on peut tout juste remarquer que, dans l'épitomé, les notices du chamois ( aľ $\alpha \alpha \gamma \rho \varsigma$ ), de la chèvre et du lièvre suivent celles de la panthêr(TIMOTHÉE DE GAZA, On animals : 26-28). Le rédacteur de la Syllogé a-t-il fait un saut du même au même lors de sa compilation? L'aigagros est déjà associé aux pardalis dans un passage précédent de l'encyclopédie emprunté à Timothée où celles-ci tendent des pièges aux chèvres sauvages pour les capturer (LAMBRos , 1885: §265, p. 93). Mais toutes ces correspondances ne peuvent expliquer cette

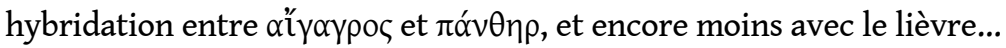

Timothée termine ce passage sur le guépard (\$281) en précisant que cet animal craint l'homme et que son origine multiple semble avoir produit sur lui une âme féminine et sans virilité. Cette interprétation " féminine » du guépard est évoquée notamment dans les traités de physiognomonie antique, que l'animal soit nommé pardalis ou panthêr ( ZUCKER, 2006 : § 16, note 38), par opposition au lion qui incarne la force virile. Là encore nous retrouvons les préjugés antiques sur l'infériorité des animaux présumés hybrides ; panthêr et leopardos ne semblent être que des demis-lions, des fauves inaccomplis ${ }^{37}$. 


\section{Conclusions} levées, bien au contraire, par le texte de la Syllogé. S'il ne nous apprend rien sur la connaissance des animaux "vrais » à Byzance, il est très riche d'informations sur les différentes strates de réception (et de confusion) des savoirs antiques sur les panthères et les guépards. Les problèmes rencontrés par Marwazī, pour interpréter et traduire en arabe le lexique grec des grands félins, démontrent la difficile appréhension de ces zoonymes aux significations mouvantes. Avec très peu de relations directes à la réalité zoologique, les panthères de Timothée forment un groupe $d$ ' « animaux philologiques ॥ $^{39}$ dont l' « habitat » reste confiné au parchemin des traités zoologiques. connaissance de l'animal dans le monde grec. Outre les éléments factuels et historiques sur l'envoi de ces animaux comme cadeau d'ambassade - enrichis par la précieuse notice de Marwazi ${ }^{40}-$, ces textes sont le réceptacle des descriptions de la morphologie si particulière de la girafe à l'époque impériale. Oppien et Héliodore avaient su observer la nature de ses cornes et sa marche à l'amble, détails extrêmement rares dans toute la littérature « zoologique » pré-linnéenne. Il n'est d'ailleurs pas exclu, comme nous l'avons $\mathrm{vu}$, que les chroniqueurs du règne de Constantin IX, décrivant la girafe qui lui fut envoyée, n'aient utilisé ces textes vieux de plusieurs siècles (ou leurs épitomés plus récents) pour conformer ce qu'ils voyaient à l'autorité des auteurs passés. 

ou de référent pour discuter du statut de l'hybridation «naturelle» entre animaux sauvages, qui semble une pratique assez fréquente, mettant en œuvre des espèces très variées, si l'on en croit le § 273. Ici la pardalis est à l'origine de la girafe et du léopard et ressemble à la panthêr. De fait, il n'est pas clairement dit que la pardalis se soit accouplée à un chameau pour faire une girafe ${ }^{42}$, de même pour la panthêr. À cause de son apparence étrange, la girafe semble être la réunion de deux espèces (\$ 271, 1. 25-26, p. 94) ${ }^{43}$. Marwazī, de son côté, évacue cette histoire d'hybridité et de mélange, alors qu'elle est bien présente dans l'épitomé $\mathrm{du} \mathrm{XI}^{\mathrm{e}}$ siècle. L'épitomateur $\mathrm{du} \mathrm{XI}{ }^{\mathrm{e}}$ siècle parle de croisement : " and it is born from the intercourse of different animals " (TIMOTHÉE DE GAZA, On animals,

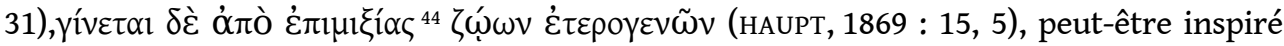
par Oppien, qui affirme lui que la girafe est née de l'union de deux espèces différentes ? Le texte de la Syllogé ne parle pas d'hybridation réelle mais de mélange ( $\mu$ íłıc) de deux animaux; mais ceci est ambigu car le contexte général est celui de l'hybridation... La sensibilité tardo-antique et médiévale était-elle plus propice à la création d'hybrides zoologiques que pendant l'Antiquité? Les descriptions antiques d'animaux exotiques et étranges fonctionnent le plus souvent comme des "puzzles ", agrégeant des parties d'animaux plus connus, pour donner une image représentable d'un animal aux formes rares et peu communes. S'ajoutant à ces assemblages de toutes sortes de bêtes, le nom luimême de ces animaux forme le plus souvent une association de deux zoonymes, ces hybrides linguistiques pouvant laisser croire à des hybridations réelles (BIVILLE, 1997) ${ }^{45}$. en elle tous les fantasmes médiévaux sur les hybridations possibles dans les contrées exotiques. "Toujours l'Afrique apporte du nouveau » ${ }^{46}$, écrivait Pline, précisant que dans les déserts les espèces différentes devaient se regrouper près des rares points d'eau, où tous les accouplements étaient possibles (PLINE, Histoire naturelle, VIII, 17, 42). La liste de ces amours étrangères et insolites, donnée au $\$ 273$ est à ce titre éloquente et spectaculaire.

Au § 281, l'origine multiple de la panthêr est donnée à la fois par les amours innombrables de sa mère et par la ressemblance de chacune des parties de son corps avec chacun des pères potentiels : la confusion entre description « puzzle » et origine hybride est ici à son comble, et pourrait bien être le fruit d'ajouts médiévaux. Mais concernant cette question difficile, il faudrait pouvoir étudier sur la durée les changements d'attitude face à l'hybridité : l'époque impériale a-t-elle été moins soucieuse de vérité zoologique que les précédentes ? La lecture « fabuleuse » du monde animal, déjà sensible chez Pline et Solin, puis « symbolique » et chrétienne à partir de la création des différentes versions grecques et latines du Physiologus(ZUCKER, 2007), puis des bestiaires médiévaux, a-t-elle favorisé l'émergence d'une sensibilité particulière face à l'hybride ?77. Il est notamment particulièrement frappant de constater que les zoonymes leopardos et leopardus sont des créations de l'époque impériale, peu après le récit par Pline de l'adultère du mâle panthère (pardus) et la lionne (voir supra), sans qu'il soit possible de savoir si ce nom nouveau servit à identifier une espèce nouvellement connue dans l'empire, peut-être le guépard (NICHOLAS, 1999 : 257-258).

L'autre point soulevé dans la Syllogé est le rapport qu'entretiennent hybridité et féminité : les fruits des amours étrangères ressemblent à leur mère et sont des animaux sans virilité. C'est le cas de la panthêr (§ 281, 1. 23-24, p. 96), qui est décrite comme craintive, et 
possédant une âme féminine et sans virilité. Mais ces "qualités » sont totalement absentes chez Marwazì : faut-il y voir là encore un ajout médiéval, ou plus sûrement l'influence des traités de physiognomonie, où la panthêr constitue un négatif de la force et du courage du lion (ZUCKER, 2006 : § 16-17, note 40)?

L'étude de la tradition et de la réception des écrits zoologiques de Timothée de Gaza s'avère complexe, faute de témoin conservé de l'œuvre originelle. Il est parfois difficile de faire la part entre copie, sélection, réécriture médiévale et éventuellement ajout d'extraits d'autres auteurs. À travers l'exemple des panthères et de la girafe, il est frappant de constater combien il peut être délicat de différencier les lectures de Timothée de celles des compilateurs médiévaux, dont la sensibilité a pu varier, notamment à propos de l'hybridité supposée des espèces exotiques. Les compilateurs arabes de Timothée ont pu eux-mêmes, comme peut-être Marwazī, "nettoyer » le texte antique de certaines conceptions zoologiques grecques, au regard de leurs sensibilités et de leurs savoirs sur l'animal. Pour s'approcher au plus près de ce qu'a pu être le texte original de Timothée, il faudrait mener une enquête comparative plus serrée entre témoins arabes et grecs, en espérant voir se développer de nouvelles collaborations entre antiquisants, byzantinistes et arabisants. Ainsi, cela permettrait de mieux connaître à la fois les textes antiques perdus et les modalités de leurs lectures et compilations médiévales, d'Orient en Occident.

\section{BIBLIOGRAPHIE}

\section{Sources}

ATTALEIATÈS Michel, Histoire, éd. Henri GRÉGOIRE, in Mélanges Rodolphe Guilland = Byzantion, 28, 1958, p. 325-419.

BUFFON, Histoire naturelle générale et particulière, avec la description du cabinet du roi, t. XIII, Paris, Imprimerie royale, 1765. [En ligne] http://gallica.bnf.fr/ark:/12148/bpt6k97502k

BUFFON, Histoire naturelle générale et particulière, servant de suite à l'histoire des animaux quadrupèdes. Supplément. Tome troisième, Paris, Imprimerie royale, 1765.

CATS BUSSEMAKER Ulco (éd.), Scholia et Paraphrases in Nicandrum et Oppianum, Paris, Firmin Didot, 1849. [En ligne] http://gallica.bnf.fr/ark:/12148/bpt6k28230s

CHASTAGNOL André (éd.), Histoire Auguste. Les empereurs romains des II ${ }^{e}$ et III ${ }^{e}$ siècles, Paris, 1994, «Bouquins».

cOSMAS Indicopleustes, Topographie chrétienne, éd. W. WOLSKA-CORNUS, Paris, 1973, « Sources

Chrétiennes, 197 ». 
DION Cassius, Histoire romaine : Cassii Dionis Cocceiani Historiarum Romanarum quae supersunt. éd. U. P. BoISSEVAIN, Berlin, Weidmann, 1901.

DION Cassius, épitomé de Xiphilin, voir Xiphilin.

ÉLIEN, La personnalité des animaux. Livres I à IX, trad. Arnaud ZUCKER, Paris, Les Belles Lettres, 2001, « La Roue à livres, 41 ».

GLYKAS Michel, Annales, éd. Immanuel BEKKER, Bonn, 1836 « Corpus scriptorum historiae byzantinae $"$.

HAUPT Moritz (éd), « Excerpta ex Timothei Gazaei libris de animalibus», Hermes, 3, 1869, p. 1-30. HÉLIODORE, Éthiopiques, éd. Robert M. RATTENBURY \& Thomas W. LuMB, trad. Jean MAILLON, Paris, 1943.

Histoire auguste (Gordien) : voir CHASTAGNOL.

Histoire auguste (Héliogabale) : voir TURCAN.

IBN MANGLĪ, De la chasse: Commerce des grands de ce monde avec les bêtes sauvages des déserts sans onde, trad. François Viré, Paris, Sindbad, 1984.

ISIDORE DE SÉvILLE, Étymologies, lib. XII (Des animaux), éd. Jacques ANDRÉ, Paris, Les Belles Lettres, 1986, « Auteurs latins du Moyen Âge».

Justinien, Corpus juridique : voir MOMMSEN et KRUGER.

KEDRENOS Georgios, Synopsis Historiôn : Georgii Cedreni Compendium historiarum, éd. Jacques-Paul MIGNE, Turnhout, 1996-1998, « Patrologia Graeca, 121-122 » [réimpr. de l'édition de 1864].

KUŠĀĞIM, Kitab al-mașāyid wa'l-mațārid, éd. Muhammad TALAS, Bagdad, 1954.

LAMBRos Spiridon (éd.), Supplementum Aristotelicum. vol. 1. Aristophanis Historiae animalium epitome II. Excerptorum Constantini de natura animalium Libri duo Aristophanis Historiae animalium epitome, subjunctis Aeliani Timothei aliorumque eclogis, Berlin, Typis et impensis G. Reimer, 1885, «Commentaria in Aristotelem Graeca ». [En ligne] http://www.archive.org/details/ supplementumari01arisgoog

AL-MANSŪR, Al-Mansur's book On Hunting, éd. Terrence CLARK et Muawiya DERHALLI, Warminster, Aris and Philipps, 2001.

MARCELlinUS COMES, Chronicon, éd. Theodor MOMMSEN, Berlin, 1894, « Monumenta Germaniae historica, 11, Chronica Minora, 2 ».

MARWAZİ : voir KRUK 2001.

MOMMSEN Theodor \& KRUGER Paul (eds), Justinian Corpus Juris Civilis. vol. I, Institutiones. Digesta, Berlin, Weidmann. 1889. [En ligne] http://www.archive.org/details/corpusjuriscivilookrueuoft MÜLLER Karl (éd.), Geographi graeci minores. I, Paris, Firmin Didot, 1855. [En ligne] http:// gallica.bnf.fr/ark :/12148/bpt6k28215w OPPIEN. La chasse, éd. Antoine-Isaac SILVESTRE DE SACY, trad. Jacques-Nicolas BELIN DE BALLU, Strasbourg, Librairie Académique, 1787.

OPPIEN, « Poème des cynégétiques », dans La pêche et la chasse dans l'Antiquité, par Oppien de Cilicie, éd. et trad. E.-J. DE BOURQUIN, Coulommiers, 1877.

OPPIEN, Cynegetica, éd. Alexander W. MAIR, Cambridge-Londres, 2002 « Loeb Classical Library, $219 »$. 
oppIEN, Cynégétiques, in Arrien et Oppien d'Apamée, L'art de la chasse, Cynégétiques, trad. Louis L'Allier, Paris, Les Belles Lettres, 2009, « La Roue à livres, 54 ».

PACHYMÈRES Georges, Relations historiques. I-II, Livres I-IV = Georgii Pachymeris relationes historicas, éd. Albert FAILLER, trad. Vitalien LAURENT, Paris, 1984.

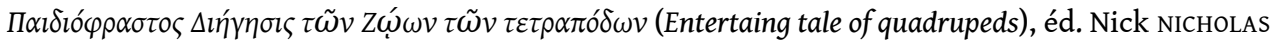
et George BALoglou, New York-Chichester, Columbia University Press, 2003.

PANTECHNÈS Constantin : voir NICHOLAS, 1999, « Appendix ».

PHILOSTORGE, Ecclesiastical History, dans The Ecclesiastical History of Sozomen... also The Ecclesiastical History of Philostorgus. éd. Edward WALFORD, Londres, 1855, « Bohn's Ecclesiastical Library ».

PHILOSTORGE, Histoire ecclesiastique. éd. Joseph BIDEZ, Leipzig, 1913, « Die griechischen christlichen Schriftsteller der ersten drei Jahrhunderte, 21 ».

PIERRE GILlES d'Albi, Ex Æliani Historia [...] De vi et natura animalium, Lyon, Sébastien Gryphe, 1535.

PIERRE GILLES d'Albi, Topographie de Constantinople : P. Gyllii de Constantinopoleos Topographia lib. IV, Lugduni Batavorum, Ex officina Elzeviriana, 1632.

PLINE, Histoire naturelle, livre VIII, éd. Alfred ERNOUT, Paris, Les Belles lettres, 1952 (réimpr. 2003).

PSELLOS Michel, Michaeli Pselli Orationes panegyricae, éd. George T. DENNIS, Stuttgart - Leipzig, Teubner, 1994, « Bibliotheca scriptorum graecorum et romanorum Teubneriana. Auctores graeci ».

Luxorius = Luxorius. A latin poet among the Vandals, éd. Morris ROSENBLUM. New York-London, Columbia University Press, 1961.

SCYLITZÈs Jean, Synopsis historiarum, éd. Ioannis THURN, Berlin - New York, 1973.

Syllogé Constantini : voir LAMBRos.

TURCAN Robert (éd.), Histoire Auguste, t. III, ${ }^{\text {re }}$ partie, Paris, Les Belles Lettres, 1993, « Collections des universités de France ».

KOPF Lothar, « The Zoological Chapter of the Kitab al-Imta' wal-Mu'anasa of Abu Hayyan al-Tauhidi (10th Century)», Osiris, 12, 1956, p. 390-466.

Scholies sur Agatharchides : voir MÜLLER.

Scholies sur Oppien : voir CATS BUSSEMAKER.

AL-TAWHĪDİ : voir KOPF.

THÉOPHANE LE CONFESSEUR, Chronographie : The Chronicle of Theophanes Confessor. Byzantine and Near Eastern history, AD 284-813, éd. et trad. Cyril MANGO, Roger SCOTT, Geoffrey GREATEX, Oxford, 1997. TIMOTHÉE DE GAZA, On animals = Peri zôôn, trad. Friedrich Simon Bodenheimer \& Alexander Rabinowitz, Paris, 1949 « Collection de travaux de l'Académie internationale d'histoire des sciences, 3 ».

TIMOTHÉE DE GAZA, Épitomé : voir HAUPT.

XIPHILINUS Johannes, Dion Cassius, L'Histoire romaine, livre Livre LXXVII (épitomé de Xiphilin), éd. E. GROS, Paris, Firmin Didot, 1866. 


\section{Études}

BIVILLE Frédérique, « Hybridations naturelles et linguistiques en zoonymie antique », in MELLET Sylvie (éd.), Les zoonymes. Actes du colloque international, Nice, 23-25 janvier 1997, Nice, 1997, «Publications de la faculté de sciences humaines de Nice, nouvelle série, 38 », p. 59-76. BODSON Liliane, « Naming the exotic animals in ancient Greek and Latin », in Animal names, (Actes du colloque international, oct. 2003), Venise, Istituto Veneto di Scienze, Lettere ed Arti, 2005, p. 453-482.

BUQUET Thierry, « La girafe, belle inconnue des bibles médiévales. Camelopardalis: un animal philologique ", Anthropozoologica, 43/2, 2008, p. 47-68. [En ligne] http://halshs.archivesouvertes.fr/halshs-00352040/fr/ (version éditeur)

BUQUET Thierry, «Le guépard médiéval, ou comment reconnaitre un animal sans nom », Reinardus 23, 2011, p. 12-47. [En ligne] http://halshs.archives-ouvertes.fr/halshs-00655131/fr/ (version auteur déposée sur HalSHS).

BUQUET Thierry, « La belle captive. La girafe dans les ménageries princières au Moyen Âge », in Beck Corinne et Guizard-Duchamp Fabrice (éds), La bête captive au Moyen Âge et à l'époque moderne. Actes du colloque de Valenciennes, 8-9 novembre 2007, Amiens, Encrage, « Encrage université », p. 65-90. [En ligne] http://halshs.archives-ouvertes.fr/halshs-00664537 (version auteur déposée sur Hal).

BUQUET Thierry, « Nommer les animaux exotiques de Baybars, d'Orient en Occident », in Mélanges Jacqueline Sublet, Beyrouth, Presses de l'Ifpo, 2012b (à paraître).

BURSTEIN Stanley M., « An Elephant for Anastasius : A Note on P. Mich. inv. 4290 », The Ancient History Bulletin, 6/2, 1992, p. 55-57.

CANNUYER Christian, La girafe dans l'Égypte ancienne et le verbe SR. Étude de lexicographie et de symbolique animalière, Bruxelles, Société Belge d'Études Orientales, Éditions Illustrata sprl, 2010, «Acta Orientalia Belgica, Subsidia, 3 ».

COHEN Marcel, «Zèbre, Zecora, Hippotigris. Aventures lexicales dans les langues romanes », Romania, 76, 1955, p. 145-182.

Divyabhanusinh Chavda, The End Of A Trail : The Cheetah In India, New Delhi - New York, Oxford University Press, 2006, « Oxford India Paperbacks ».

GATIER Pierre-Louis, « Des girafes pour l'empereur », Topoï : Orient-Occident, 6/2, 1996, p. 903-941. GATIER Pierre-Louis, «Les girafes de Gaza », in SALIou Catherine (éd.), Gaza dans l'Antiquité tardive : histoire, rhétorique, archéologie. Actes du colloque international de Poitiers (6-7 mai 2004), Salerno, Hélios, 2005, p. 75-92.

ISKANDAR Albert Z., « A Doctor's Book on Zoology : al-Marwazi's Taba'i' al-hayawan (Nature of Animals) Re-Assessed », Oriens, 27, 1981, p. 266-312. [En ligne] http://www.jstor.org/pss/1580569 KRUK Remke, «Timotheus of Gaza's On animals in the arabic tradition », Le Museon, 114/3-4, 2001, p. 355-387.

KRUK Remke, «Zarafa : Encounters with the Giraffe, from Paris to the Medieval Islamic World », ingRUENDLER Beatrice et COOPERSON Michael (éds), Classical Arabic Humanities in their Own Terms. Festschrift for Wolfahrt Heinrichs on his 65th Birthday, Leiden, Brill, 2008, p. 568-592. 
MORGAN John R., « Two Giraffes Emended », The Classical Quarterly, 38/1, 1988, p. 267-269.

[En ligne] http://www.jstor.org/stable/639235

NICHOLAS Nick, « A conundrum of cats : pards and their relatives in Byzantium », Greek, Roman and Byzantine Studies, 40/3, 1999, p. 253-298.

ŠEVČEnKo Nancy P., « Wild Animals in the Byzantine Park », in LitTlewood Anthony, MAGUiRE Henry, woLSCHKE-BULMAHN Joachim (éds), Byzantine Garden culture, Washington, Dumbarton Oaks, 2002, p. 69-86.

STEIER August, « Timotheos von Gaza », Paulys realencyclopädie der classischen Altertumwissenschaft, Timon-Tribus, Munchen, Alfred Duckenmüller Verlag, 1937, col. 1339-1341.

THOMPSON D’Arcy W., «The Greek for a Zebra », The Classical Review, 57/3, 1943, p. 103-104.

[En ligne] http://www.jstor.org/stable/702479.

VINCENT Thierry, "Quand les guépardiers rivalisaient de savoir-faire avec les fauconniers ", La chasse au vol au fil des temps. Exposition présentée et organisée par le Musée international de la chasse, Gien, 5 juin-23 octobre 1994, Gien, Musée international de la chasse, 1994, p. 153-162.

VIRÉ François, « Fahd », Encyclopédie de l'islam (EI²), t. II (C-G), Leiden, Brill, 1977, p. 757-761.

VIRÉ François, « Namir-Nimr », Encyclopédie de l'islam (EI²), t. VII. MIF-NAZ, Leiden, Brill, 1993, p. 947-950.

WOTKE Friedrich, JEREH Helga, « Panther », Real-Encyclopädie de classischen Altertumswissenschaft (Pauly-Wissova), 18/2b, Stuttgart, Dreckenmüller, 1949, col. 747-778.

ZUCKER Arnaud, «La physiognomonie antique et le langage animal du corps », in Le modèle animal. I. Actes du XXXVIII Congrès international de l'APLAES : " L'animal, un modèle pour l'homme » = Rursus, 1, 2006. [En ligne] http://rursus.revues.org/58

ZUCKER Arnaud, « Morale du Physiologos : le symbolisme animal dans le christianisme ancien (II ${ }^{\mathrm{e}}-\mathrm{V}^{\mathrm{e}}$ s.) », Rursus, 2, 2007 (Le modèle animal, II). [En ligne] http://rursus.revues.org/142

ZUCKER Arnaud, «Qu'est-ce qu'une paraphrasis ? L'enfance grecque de la paraphrase », Rursus, 6, 2011 (Relire, récrire, prolonger. Adaptations gréco-latines). [En ligne] http://rursus.revues.org/476

\section{NOTES}

1. Je tiens à remercier chaleureusement mes collègues Jacques-Hubert Sautel (IRHT), Justine Gaborit et Frédéric Alpi (Ifpo) pour l'aide précieuse qu'ils m'ont apportée pour la traduction et la révision des textes grecs.

2. L'information principale vient de Suidas ( $I X^{e}$ siècle), qui nous dit que Timothée écrivit quatre livres sur les quadrupèdes, les oiseaux et les serpents d'Inde, de Lybie et d'Égypte, dans un style épique (TIMOTHÉE DE GAZA, On animals, 4-5)

3. Contrairement à ce qui est indiqué dans l'introduction de Bodenheimer et Rabinowitz, ce résumé est un épitomé (résumé, abrégé) et non une paraphrase (ZUCKER, 2011 : note 52).

4. Les renvois à Timothée signalés dans notes de l'éditeur L. Kopf ne sont pas très convaincants ; il semble que Tawhīdī utilise un savoir zoologique grec assez courant dans le monde arabe, sans qu'il soit certain de l'identifier à tel ou tel auteur (KRUK, 2001: 356). Il m'a été impossible d'identifier dans l'édition de Kopf un emprunt certain à Timothée de Gaza. Ainsi il m'a semblé préférable, dans le cadre de cette étude sur la Syllogé Constantini, de ne pas utiliser cette source. 


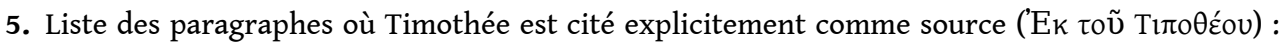
$291,302,315,340,363,385,388,401,421,430,466,507,566$. Un index des auteurs cités par paragraphe est donné par Lambros dans son introduction (LAMBRos, 1885 : XVI-XVIII)

6. Nous reviendrons plus loin sur l'identification zoologique de ces zoonymes grecs, dont les deux derniers posent d'évidentes difficultés.

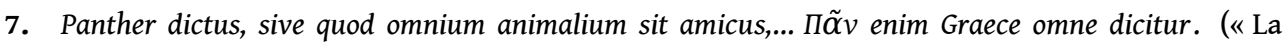
panthère se nomme ainsi parce-qu'elle est l'amie de tous les animaux... "Pân" signifie en grec "tout" ").

8. Il n'existe pas de panthère à queue courte; les seuls félins à queue courte appartiennent à la famille des lynx et sont de taille très inférieure à celle des panthères. On a pu croire qu'Oppien désignait ici la différence entre la panthère et le guépard, ce dernier ayant une corpulence moindre que celle de la panthère ; le guépard a effectivement une queue très longue, qui lui sert de balancier pour changer rapidement d'orientation dans ses courses très rapides, mais l'appendice de la panthère est également très long.

9. Il faut noter que Remke Kruk n'a visiblement pas consulté la Syllogé Constantini dans son étude.

10. Sur la différentiation faite par les Arabes entre panthère et guépard, voir F. VIRÉ, Encyclopédie de l'Islam, 2e éd., 1977 (fahd) et 1993 (namir-nimr).

11. La première mention de pardis mansuetis («pards» apprivoisés, sans doute des guépards), dressés pour la chasse, qui est évoquée par le poète Luxorius, date du règne du roi vandale Tharasmond (496-523), à Carthage (LUXoRIUs, poème 74, De pardis mansuetis qui cum canibus venationem faciebant, p. 154-156).

12. Chez ces deux auteurs, il s'agit de la panthère (namir ou nimr), ce qui correspondrait d'avantage à l'utilisation d'Oppien (pardalis).

13. Ce passage correspond très bien à l'épitomé du XI ${ }^{\mathrm{e}}$ siècle (chap. 11,5)

14. Chez Élien, la panthère utilise aussi cette bonne odeur pour attirer ses proies (Personnalité des animaux, $\mathrm{V}, 40$ ).

15. Il faut rappeler que la panthère noire ne constitue pas une espèce propre : il s'agit d'une mutation génétique provoquant la couleur très sombre du pelage, sur lequel on peut discerner quand même les taches caractéristiques de la panthère.

16. Races aujourd'hui disparues, comme presque toutes celles d'Asie. Seul le guépard africain sub-saharien survit encore (DIVYABHANUSINH, 2006, 173-179).

17. Le dessin de cet éléphant pourrait être reconnu sur un papyrus tardo-antique (BURSTEIN, 1992), hypothèse contestée par GATIER (1996: 920, note 101).

18. «India Anastasio principi elephantum, quem Plautus poeta noster lucabum nomine dicit, duasque camelopardalas pro munere misit. » (GATIER, 1996 : 919, note 97).

19. La lecture de ce passage peu clair en arabe est peut-être fautive et pourrait plutôt donner : « leurs queues étant noires à l'extrémité » (KRUK, 2008 : 575, note 25), ce qui correspond bien à la physionomie de l'animal.

20. Bodenheimer n'est pas très sûr de sa traduction : "One could also translate: "a man (coming) from India"! » (TIMOTHÉE DE GAZA, On animals : 31, note 4).

21. Sur le nom de cette ville et le rôle du port d'Aila, voir remarques et bibliographie chez GATIER 1996 : 920, note 100

22. Il est tout à fait remarquable que cette notice sur le zèbre ne soit pas reprise ni dans la Syllogé Constantini, ni par Marwazī. Je reviendrai plus loin sur cette absence, ainsi que celle du tigre dans la Syllogé, pourtant présent chez Marwazī (KRUK, 2001 : 368-369). Le tigre est présent dans le nom grec du zèbre : hippotigris, « cheval-tigre » (COHEN, 1955, THOMPSON, 1943).

23. L'édition anglaise de Mair dit que l'asymétrie des membres fait qu'elle semble accroupie (p. 152-153). De Bourquin (1877: 207-208) traduit comme suit: « [la différence de hauteur des 
trains avant et arrière] introduit une sorte de claudication dans l'allure de l'animal ». Belin de Ballu, au XVIII ${ }^{\mathrm{e}}$ siècle, interprète dans ses notes ce passage comme la description « quoique d'une manière obscure » de la démarche de la girafe, sans nommer l'amble (1787 : 81-82 et note sur la ligne 20, p. 201). Il s'appuie sur Héliodore et Pierre Gilles (PIERRE GILLES D'ALBI, 1535 : livre V, chap. 23, p. 159-160), ce dernier décrivant une marche boiteuse, ceci influençant la traduction de Belin de Ballu.

24. P.-L. Gatier affirme pourtant « Ce qu'il en dit [de la girafe], comme de bien d'autres animaux, n'est que le reflet des Cynégétiques du pseudo-Oppien. » (GATIER, 1996 : 912, note 58).

25. P.-L. Gatier dit un peu vite que la description de Philostorge n'apporte aucune nouveauté par rapport aux autres textes antiques sur la girafe (GATIER, 1996 : 912, note 57).

26. Zoonyme obscur mentionné non seulement par Philostorge mais aussi par Cosmas Indicopleustes (Topographie chrétienne, XI, 3, éd. Wolska-Cornus: 320), par Théophane le Confesseur (Chronographie: 170), puis au XII ${ }^{\mathrm{e}}$ siècle par Georgios Kedrenos (Synopsis Historiôn, I, 564). À la Renaissance, Pierre Gilles d'Albi corrigera dans le texte de Kedrenos les taurelephantos en taurelaphoi (Topographie de Constantinople : chap. 25, p. 189-190).

27. Autres mentions du même événement chez Jean Scylitzès (Synopsis historiarum, Constantin Monomaque, 28, p. 475) et Michel Psellos (Orationes panegyricae, I, 245-289).

28. Le zèbre a été repris dans l'épitomé de Jean Xiphilinus ( $\mathrm{XI}^{\mathrm{e}}$ siècle) sur Dion Cassius $(77,6,2$ :

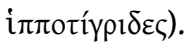

29. Sur le zèbre : THOMPSON, 1943 ; COHEN, 1955 et BUQUET, 2012 b.

30. Ignace, Épître aux Romains, 5, 1, cité par NICHOLAS, 1999 : 279.

31. Dans les deux passages, des leopardi et des lions apprivoisés, dressés par des dompteurs, étaient chargés d'effrayer les invités d'Héliogabale pour son amusement. Il est également fait mention de leopardi mansueti pour le règne de Gordien (CHASTAGNOL, 1994 : Gordien, III, 33, 1, p. 740-743).

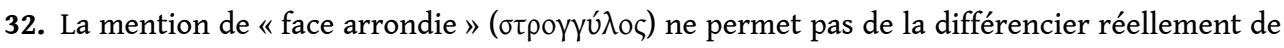
celle de la panthère; le pseudo-Aristote est plus exact en disant qu'elle est petite ( $\mu$ ikpó Physiognomonie, 810 a 39 cf. ZUCKER, 2006 : note 53) ; de faite celle de la panthère est beaucoup plus forte et massive.

33. Manuscrit $D$, signalé par LAMBRos ( $1885: 96$, note sur la ligne 7 ).

34. Outre le lexique de la langue arabe qui sépare bien les deux espèces ( $f a h d$ et namir), les savoirs zoologiques arabes médiévaux définissent sans ambiguïté les différences anatomiques entres ces animaux (VIRÉ, 1977 et 1993).

35. J'ai évoqué ces difficultés d'identification du lexique de la panthère et des guépards pour le Moyen Âge occidental dans mon article «Le guépard médiéval, ou comment reconnaître un animal sans nom » (BUQUET, 2011).

36. Chez Timothée de Gaza, et dans le traité de physiognomonie de Polémon (zUCKER, 2006, note 53).

37. Isidore de Séville dit que l'union de la lionne et de la panthère, le leopardus, est un être dégénéré, comme le mulet ou le bardot (Étymologies, 12, 2).

38. Alors que les sources byzantines sont peu bavardes sur les guépards des ménageries ( NICHOLAs, 1999), ces animaux ont été vus et identifiés par des voyageurs étrangers qui les ont vu aux spectacles organisés à l'hippodrome : Marwazī et Benjamin de Tudèle au XII ${ }^{\mathrm{e}}$ siècle (šEVČENKO, $2002: 75-76)$.

39. J'ai introduit cette notion à propos de la girafe pour l'Occident médiéval, où la camelopardalis de Pline et d'Isidore de Séville n'a jamais été confondue avec les quelques girafes "vraies » présentes en Europe avant le XV $\mathrm{XV}^{\mathrm{e}}$ siècle (BUQUET, 2008). 
40. Il faut préciser que Marwazī connaissait bien les girafes pour avoir pu en observer un spécimen vivant à Ispahan en l'an de l'hégire 474 (1081-1082) à la demande du vizir Malik-Shāh ( ISKANDAR, $1981: 272$, note 30 ).

41. Contrairement à ce qu'affirme P.-L. GATIER (1996 : 912-913).

42. Déjà Strabon mettait en doute la possible hybridation entre le chameau et la panthère, expliquant que les girafes ne ressemblent en rien aux panthères et que les taches de leur pelage ont plus à voir avec celles du faon (BODson, 2005 : 471-472)

43. On trouve la même remarque dans les scholies sur Oppien (CATS BUSSEMAKER, $1849: 256, \mathrm{n}^{\circ}$ 462): "giraffes: a natural monster and altogether a wonder, somehow uniting two species in one » (BODSON, $2005: 469$, note 94 ).

44. $\dot{\varepsilon} \pi \iota 1 \xi i ́ \alpha$ : relation mutuelle, commerce. Sens moderne : « métissage ».

45. Les thèses de F. Biville ont été débattues par L. Bodson qui, réfutant l'idée des hybridations zoonymiques, a proposé un système propre à la langue grecque pour nommer les animaux exotiques (BODSON, 2005).

46. Semper aliquid novi Africam adferre. Pline dit qu'il cite un proverbe grec (unde etiam vulgare Graeciae dictum)

47. Il faut noter que les articles de F. BIVILLE (1997) et de L. BoDson (2005) ne mettent pas en perspective cette possible évolution dans le temps de la perception des "hybrides exotiques» entre période classique et époque impériale.

\section{RÉSUMÉS}

L'article traite de la transmission de l'œuvre zoologique perdue de Timothée de Gaza (fin V ${ }^{\mathrm{e}}$ début $\mathrm{VI}^{\mathrm{e}}$ siècle après $\mathrm{J} . \mathrm{C}$.) dont des passages ont été restitués dans la compilation byzantine d'histoire naturelle, le Sylloge Constantini daté $\mathrm{du} \mathrm{x}^{\mathrm{e}}$ siècle, et dont nous étudions les paragraphes 260-282. Ces extraits décrivent plusieurs sortes de panthères (pardalis, leopardos, panther) et la girafe (kamelopardalis), dans un contexte évoquant l'hybridation entre races différentes, produisant des animaux à la nature mixte. Il s'agit d'essayer de reconstituer les passages perdus de Timothée à propos de ces animaux, en s'appuyant également sur leur compilation par un auteur arabe du XII ${ }^{\mathrm{e}}$ siècle, Marwazī, qui donne notamment pour la girafe des informations absentes dans la Syllogé et dans l'épitomé byzantin de Timothée rédigé au XI ${ }^{\mathrm{e}}$ siècle. À travers l'étude de ces différents extraits, l'article évoque la réception médiévale de l'hybridité supposée de certaines espèces exotiques et l'identification et la différenciation entre guépard, panthère et léopard dans le monde grec.

This paper deals with the transmission of the lost zoological work of Timotheus of Gaza (end of the 5th century, beginning of the 6th century AD), of which some passages have been preserved in a zoological Byzantine compilation, the Sylloge Constantini, dating from the 10th century, and of which we study the paragraphs 260-282. These extracts describe several species of panthers ( pardalis, leopardos, panther) and the giraffe (kamelopardalis), in a context of hybridization between different species, producing crossbreed mixture animals. This paper attempts to identify lost Timotheus' excerpts, relying on their compilation by the Arabic author Marwazì (12th century), which gives, about the giraffe, further information missing in the Sylloge and in the Byzantine Timotheus' epitome written in the 11th century. Throughout the study of these different extracts, 
the paper investigates the mediaeval reception of the presumed hybridity of some exotic species and the identification and the distinctness between cheetah, panther and leopard in the Greek world.

\section{INDEX}

Keywords : Timothy of Gaza, giraffe, cheetah, panther, leopard, philology, Marwazī, hybrid, epitome, excerpta, Sylloge Constantini

Mots-clés : Timothée de Gaza, girafe, guépard, panthère, léopard, philologie, Marwazī, hybride, épitomé, excerpta, Syllogé Constantini

\section{AUTEUR}

\section{THIERRY BUQUET}

Institut français du Proche-Orient, Diplômé de l'EPHE en histoire et philologie, Thierry Buquet est ingénieur au CNRS, rattaché à l'Institut français du Proche-Orient (Ifpo, Damas), après avoir travaillé pendant quinze ans à l'Institut de recherche et d'histoire des textes (IRHT, Orléans). Il est aujourd'hui responsable-médiateur des ressources électroniques de l'Ifpo. Ses recherches portent sur l'histoire des animaux exotiques au Moyen Âge, d'Orient en Occident, principalement la grande faune africaine et asiatique (girafe, éléphant, panthère, guépard, lion, zèbre, etc.). Il s'intéresse à l'histoire des ménageries princières, à l'histoire des savoirs zoologiques et leur transmission de l'Antiquité à la Renaissance, ainsi qu'à l'histoire des zoonymes.

Page personnelle (Ifpo) : http://www.ifporient.org/thierry-buquet 